\title{
Perioperative outcomes of bipolar energy instruments in total laparoscopic hysterectomy
}

\author{
Yakup Yalcin ${ }^{1,2}$, Serenat Eris Yalcin ${ }^{3}$ \\ ${ }^{1}$ Department of Obstetrics and Gynecology, Istinye University School of Medicine, Istanbul, Turkey \\ ${ }^{2}$ Department of Gynecologic Oncology, Medical Park Hospital, Antalya, Turkey \\ ${ }^{3}$ Department of Obstetrics and Gynecology, Suleyman Demirel University School of Medicine, Isparta, Turkey
}

\begin{abstract}
Objectives: To compare conventional and advanced bipolar energy instruments in terms of perioperative outcomes in patients who underwent total laparoscopic hysterectomy (TLH).

Material and methods: The data of 101 patients who underwent TLH between June 2017 and December 2018 for benign gynecological disorders were analyzed retrospectively. Conventional bipolar forceps (Robi forceps) were used in 37 patients and advanced bipolar instruments (LigaSure) were used in 64 patients. Data about the characteristics of the patients, operation time, estimated blood loss, length of hospital stay and other perioperative outcomes were compared.

Results: The mean ages of the patients in the conventional bipolar and LigaSure groups were $47.6 \pm 6.5$ and $48.1 \pm 7$ years, respectively $(p>0.05)$. There was no statistically significant difference between the two groups with regard to all other patient characteristics; body mass index, parity, previous pelvic operation and indications of hysterectomy $(p>0.05)$. The mean operation time ( $41 \pm 13.2$ vs $37 \pm 11.5 \mathrm{~min}$ ), estimated intraoperative blood loss ( $70 \pm 22$ vs $65 \pm 21 \mathrm{~mL}$ ) and absolute change in hemoglobin $(-1.23 \pm 1.12$ vs $-1.11 \pm 1.14 \mathrm{~g} / \mathrm{dL}$ ) were slightly higher in the conventional bipolar group. However, there was no statistical significance with respect to these differences between the groups $(p>0.05)$.

Conclusions: Our findings indicate that a conventional bipolar system is as safe and effective as LigaSure, and it may be used as an alternative option for patients undergoing TLH in low-income hospitals.
\end{abstract}

Key words: conventional bipolar instrument; LigaSure; vessel sealing; total laparoscopic hysterectomy

Ginekologia Polska 2019; 90, 11: 640-644

\section{INTRODUCTION}

Hysterectomy is one of the most frequently performed gynecologic surgical procedures and may be performed by abdominal, vaginal and laparoscopic approach [1]. Total laparoscopic hysterectomy (TLH) has been performed increasingly over the years due to less postoperative pain, less risk of incision infection, reduced adhesion formation, shorter recovery time and early discharge. It has now become an indispensable part of gynecology practice [2]. Although TLH is superior to abdominal hysterectomy, the vaginal approach still preserves its current value in most benign indications.

Conventional electrosurgery comprises two types of diathermy as unipolar and bipolar. In monopolar electrosurgery, high voltage is used for cutting, dissection and fulguration. In bipolar electrosurgery, low voltage is used for coagulation [3]. The basic working principle of the conven- tional bipolar energy system is to denature the collagen and elastin inside the vessel wall or tissue by providing electrical energy between the two jaws. Since the electrical current is present between the two jaws, a neutral electrode is not required and surgical safety is higher [4].

There have been improvements in surgical instruments as laparoscopic operations have increased over time. Advanced vascular sealing devices that work with ultrasonic and bipolar energy have been developed [5]. These novel advanced bipolar vessel-sealing devices appear to decrease the lateral thermal spread remarkably; thus they are safe and time-efficient in comparison to traditional bipolar electrosurgical devices. These beneficial features show up more evident in difficult procedures $[6,7]$. LigaSure is a hemostatic device developed as an advanced bipolar energy system (Covidien-Valleylab today Medtronic-Covidien, Boulder, CO, USA) and has demonstrated efficacy in a variety of surgical 
procedures [8]. LigaSure denatures collagen and elastin in the vessel wall and sticks with high mechanical pressure. Then the vessel is cut into two parts with the help of the knife inside of LigaSure. It was proven that it can be safely used in vessels up to $7 \mathrm{~mm}$ [8]. LigaSure has been shown to be on a similar level in terms of efficiency in comparison to clips, sutures and ultrasonic vessel sealing methods [9].

In this study, we aimed to compare conventional and advanced bipolar energy instruments in terms of perioperative outcomes in patients who underwent total laparoscopic hysterectomy.

\section{MATERIAL AND METHODS}

In this study, the data of 101 patients who underwent TLH between June 2017 and December 2018 for benign gynecological disorders at the Isparta City Hospital were analyzed retrospectively. Conventional bipolar forceps (Robi forceps, Karl Storz, Tuttlingen, Germany) were used in 37 patientsand the LigaSure $5 \mathrm{~mm}$ device (Medtronic-Covidien, Boulder, CO, USA) that is an advanced bipolar instrument was used in 64 patients. Initially, there was only a conventional bipolar device in our hospital because of financial insufficiency. Nevertheless, we performed the procedure on the first 37 patients with this device. After LigaSure was supplied, we performed the procedure on the other cases with this instrument. Patient characteristics, including age, body mass index (BMI), parity, surgical indication, previous pelvic surgery history and perioperative results were obtained from the patients' medical records. The perioperative results included uterine weight, operation time, estimated blood loss, blood transfusion, length of hospital stay and intraoperative complications. The eligibility criteria included patients who required hysterectomy for benign conditions such as symptomatic uterine fibroids, abnormal bleeding or other benign diseases. The exclusion criteria were the current pregnancy status and malignant diseases of the genital tract. All patients were managed with the same protocol for preoperative and postoperative treatments. We also administered $1 \mathrm{~g}$ of cephalosporin antibiotic intravenously 30 minutes before the incision for prophylaxis. All surgeries were performed by the same surgeon.

\section{Surgical Technique}

All operations were performed under general anesthesia in a dorsal lithotomy and Trendelenburg position. In all patients, the stomach was decompressed with a nasogastric tube and the catheter was inserted into the bladder before the surgery. Depending on the diameter of the cervix and the size of the uterus, the appropriate vaginal manipulator was inserted into the uterine cavity. A VCare uterine manipulator (Conmed, NY, USA) was used in the operations. The surgical procedures were performed through 3 or
4 laparoscopic trocars: a 10-mm trocar for the camera from the umbilicus, one 5-mm lateral trocar at $2 \mathrm{~cm}$ superior of the left iliac spina and one 5-mm suprapubic trocar for the laparoscopic instruments. In some cases, a fourth 5-mm trocar was inserted from the right lower quadrant if it was needed. The surgeon was on the left side of the patient and performed the operation using the left and midLine trocars. All operations were performed with Robi forceps and Metzenbaum scissors or LigaSure for sealing, dissection and hemostasis. The vaginal cuff was closed by intracorporal suturing using a polyglactin 910 suture that is a late absorbable material (Vicryl, Ethicon, Johnson \& amp; Johnson Medical Devices Companies, USA). All laparoscopic procedures were performed without conversion to laparotomy.

Operation time was defined as the time from the initial skin incision to closure of the abdominal trocar incisions. Estimated blood loss in the operation was calculated by subtracting the given amount of fluid from the total outcome in the suction unit. The position of the patients was the reversed Trendelenburg position to obtain all intra-abdominal fluid. Furthermore, quantitative blood loss was calculated by comparing the preoperative hemoglobin value to the hemoglobin value on the first day after surgery.

Patients who had spontaneous micturition, gas outflow and stable vital signs were discharged on the first or second postoperative day.

\section{Statistical analyses}

The variables are presented with frequencies and mean \pm standard deviation values. Differences between two groups were analyzed using unpaired $t$-test, chi-squared $\left(X^{2}\right)$ test and Fisher's exact test. $P<0.05$ was considered statistically significant. Statistical analyses were performed in SPSS 24.0 (IBM Corporation, Armonk, NY).

\section{RESULTS}

The data of a total of 101 patients were analyzed. The mean ages of the patients in the conventional bipolar and LigaSure groups were $47.6 \pm 6.5$ and $48.1 \pm 7$ years, respectively $(p>0.05)$. There was no statistically significant difference between the two groups with regard to all other patient characteristics; body mass index, parity, previous pelvic operation and indications of hysterectomy $(p>0.05)$ (Tab. 1).

The mean operation time ( $41 \pm 13.2$ vs $37 \pm 11.5 \mathrm{~min})$, estimated intraoperative blood loss (70 $\pm 22 \mathrm{vs} 65 \pm 21 \mathrm{ml}$ ) and absolute change in hemoglobin $(-1.23 \pm 1.12 \mathrm{vs}-1.11 \pm 1.14 \mathrm{~g} / \mathrm{dL})$ were slightly higher in the conventional bipolar group. However, there was no significance with respect to these differences between the groups ( $p>0.05$ ) (Tab. 2).

Blood transfusion was given in one patient in each group, but the reason was not major vessel injury. One intraoperative major complication occurred in the LigaSure 
Table 1. Demographic characteristics of patients

\begin{tabular}{|c|c|c|c|}
\hline & Conventional bipolar surgery ( $n: 37$ ) & LigaSure (n: 64) & $\mathbf{p}$ \\
\hline Age (years) (range) & $47.6 \pm 6.5(39-78)$ & $48.1 \pm 7.1(40-81)$ & 0.76 \\
\hline BMI (kg/m²) (range) & $29 \pm 5.3(22-47)$ & $30 \pm 6.2(23-45)$ & 0.64 \\
\hline Parity (n) (range) & $1.8 \pm 1.3(0-7)$ & $1.7 \pm 1.1(0-5)$ & 0.84 \\
\hline $\begin{array}{l}\text { Indication } \\
\text { Uterine myoma, } \mathrm{n}(\%) \\
\text { Abnormal uterine bleeding, } \mathrm{n}(\%) \\
\text { Endometriosis, } \mathrm{n}(\%) \\
\text { Other benign pathologies, } \mathrm{n}(\%)\end{array}$ & $\begin{array}{l}19(51.3) \\
8(21.6) \\
4(10.8) \\
6(16.3)\end{array}$ & $\begin{array}{l}34(53.1) \\
12(18.7 \\
5(7.9) \\
13(20.3)\end{array}$ & $\begin{array}{l}0.61 \\
0.23 \\
0.14 \\
0.11\end{array}$ \\
\hline Previous cesarean sections, n (\%) & $9(24.3)$ & $14(21.8)$ & 0.44 \\
\hline
\end{tabular}

$\mathrm{BMI}$ - body mass index

Table 2. Perioperative outcomes of the two groups

\begin{tabular}{|l|l|l|l|}
\hline & Conventional bipolar surgery (n: 37) & LigaSure (n: 64) \\
\hline Uterine weight (gr) (range) & $358 \pm 261(70-820)$ & $372 \pm 284(85-940)$ \\
\hline Operation time (min) (range) & $41 \pm 13.2(30-75)$ & $37 \pm 11.5(25-65)$ \\
\hline Intraoperative blood loss (ml) (range) & $70 \pm 22(10-450)$ & $65 \pm 21(10-410)$ \\
\hline Preoperative hemoglobin (g/dL) (range) & $12.53 \pm 1.42(9.2-14.3)$ & $12.77 \pm 1.59(9.0-15.1)$ \\
\hline Postoperative hemoglobin (g/dL) (range) & $11.28 \pm 1.38(8.8-13.7)$ & $11.47 \pm 1.53(8.7-14.2)$ \\
\hline Absolute change in hemoglobin(g/dL) (range) & $-1.23 \pm 1.12(0.3-2.1)$ & $-1.11 \pm 1.14(0.2-2.7)$ \\
\hline Blood transfusion, $n$ (\%) & $1(2.7)$ & $1(1.6)$ \\
\hline Hospital stay (day) (range) & $2.2 \pm 0.8(1-3)$ & 0.18 \\
\hline TLH + BS, $n$ (\%) & $14(37.9)$ & $0.22 \pm 0.9(1-5)$ \\
\hline TLH + BSO/USO, $n$ (\%) & $23(62.1)$ & $23(35.9)$ \\
\hline Major complication, $n(\%)$ & $0(0.0)$ & $41(64.1)$ \\
\hline
\end{tabular}

TLH — total laparoscopic hysterectomy; USO — unilateral salpingo-oopherectomy; BSO — bilateral salpingo-oopherectomy; BS — bilateral salpingectomy

group. No bladder, major vessel or ureteric injury occurred in the two groups.

\section{DISCUSSION}

In this study, we investigated the differences in operative time, blood loss and other perioperative outcomes for LigaSure and conventional bipolar devices during total laparoscopic hysterectomy for benign gynecological indications.

Recently, electrosurgical sources for tissue preparation and vascular sealing have been expanded to include devices that can offer both sealing and cutting. These devices do not need to be replaced during the operation; they also resist high intraluminal pressure and have optimal coagulation properties [9]. LigaSure is an advanced bipolar device that is capable of cutting and sealing. It is able to seal vessels up to $7 \mathrm{~mm}$ in diameter and withstands up to threefold the normal systolic blood pressure. It has a minor thermal spread that is up to $4.0 \mathrm{~mm}[10,11]$. LigaSure has been successfully used in gynecologic operations, in addition to a variety of laparoscopic procedures, urologic and abdominal surgeries [12-14].
In the literature, variable results have been found about duration of operation in gynecologic or other abdominal surgeries for conventional and advanced bipolar devices [3, 15-20]. Many studies reported that LigaSure has a shorter operative time than conventional bipolar instruments. The reason for this increase in time was changing of the instruments for sealing and cutting during the operation in conventional device groups [16-18]. Additionally, the current delivered with LigaSure takes up to 7 seconds to achieve homeostasis [21]. In contrast to the literature, there was no significant difference with respect to operating time between the two groups in our study. If both conventional bipolar devices and scissors are used with both hands at the same time effectively and correctly, no difference in operation time may be expected.

Previous studies reported that intraoperative blood loss was lower in advanced bipolar groups in comparison to conventional groups $[20,21]$. According to our results, the estimated and quantitative blood loss was similar in both groups. The discrepancy between hemoglobin decrease and estimated blood loss may be thought to be due to 
postoperative hemodilution. Conventional bipolar devices and scissors may not increase the amount of bleeding when used properly. Hemostasis may be successfully achieved in both devices.

There is a doubt about the thermal spread of energy-based devices. The lateral thermal spread of electrosurgery devices may be a risk factor for major complications during laparoscopic hysterectomy. Therefore, adjacent tissues should be checked, and traction should be made while sealing and cutting during the operation. The major complication rate during laparoscopic hysterectomy is reported to be in the range from $4 \%$ to $9 \%$ in the literature [22]. In our study, this rate was $1.6 \%$, and one intraoperative complication occurred in the LigaSure group. However, the complication was not associated with the type of instruments used for sealing in the operation. This 48-y-old patient had chronic pelvic pain and history of endometriosis. Dense adhesions and an endometriotic nodule were present between the rectum and the uterus. The rectum was injured during resection of the endometriotic nodule from the rectovaginal space. We repaired this injury via laparoscopy without conversion to laparotomy. This patient recovered without complications and was discharged on the 5th postoperative day.

In studies, the length of hospital stays in patients who underwent laparoscopic hysterectomy with conventional bipolar devices was longer than those with advanced bipolar devices. Prolonged hospital stay may be caused by major complications $[19,23]$. In this study, both groups had a similar length of hospital stay following the operations. No significant difference was observed in the durations of hospital stay, because the major complication rate was the same in both groups.

The limitations of our study were the small number of patients included and its retrospective nature. Although the number of patients was not equal and the excess in the LigaSure group may seem to affect the results, the findings were not influenced because the data belonged to a single surgeon experienced in both devices. In the future, more large-scale, prospective and randomized studies will be required.

In conclusion, advanced bipolar instruments have the advantages that are closing of vascular structures in addition to their cutting capabilities, so that the process can proceed without changing instruments between hemostatic and cutting devices. In the hands of experienced surgeons, both scissors and conventional devices may be used together and there is no need to change. Thus, there is no significant difference with respect to operating time and blood loss between the two instruments. LigaSure is a disposable energy source and more expensive per case than conventional reusable energy sources. Conventional bipolar energy devices are more cost-effective due to possibility of re-use. They may be used safely and effectively in low-income hospitals that cannot access advanced bipolar energy instruments such as LigaSure.

\section{REFERENCES}

1. ACOG Committee Opinion No. 444: choosing the route of hysterectomy for benign disease. Obstet Gynecol. 2009; 114(5): 1156-1158, doi: 10.1097/AOG.0b013e3181c33c72, indexed in Pubmed: 20168127.

2. Kluivers KB, Opmeer BC, Geomini PM, et al. Women's preference for laparoscopic or abdominal hysterectomy. Gynecol Surg. 2009; 6(3): 223-228, doi: 10.1007/s10397-008-0455-1, indexed in Pubmed: 20234838.

3. Wang CJ, Yuen LT, Yen CF, et al. Comparison of the efficacy of the pulsed bipolar system and conventional bipolar electrosurgery in laparoscopically assisted vaginal hysterectomy. J Laparoendosc Adv Surg Tech A. 2005; 15(4): 361-364, doi: 10.1089/lap.2005.15.361, indexed in Pubmed: 16108737.

4. Ayroza P, Abdalla E. Manual of Gynecological Laparoscopic Surgery: Use of Electricity in Laparoscopy, 2nd ed. Tuttlingen, Germany: Endo Press. ; 2010: 43.

5. Harold KL, Pollinger $\mathrm{H}$, Matthews BD, et al. Comparison of ultrasonic energy, bipolar thermal energy, and vascular clips for the hemostasis of small-, medium-, and large-sized arteries. Surg Endosc. 2003; 17(8): 1228-1230, doi: 10.1007/s00464-002-8833-7, indexed in Pubmed: 12799888.

6. Alkatout I, Schollmeyer T, Hawaldar NA, et al. Principles and safety measures of electrosurgery in laparoscopy. JSLS. 2012; 16(1): 130-139, doi: 10.4293/108680812X13291597716348, indexed in Pubmed: 22906341.

7. Richter S, Kollmar O, Schilling MK, et al. Efficacy and quality of vessel sealing: comparison of a reusable with a disposable device and effects of clamp surface geometry and structure. Surg Endosc. 2006; 20(6): 890894, doi: 10.1007/s00464-005-0380-6, indexed in Pubmed: 16738977.

8. Landman J, Kerbl K, Rehman J, et al. Evaluation of a vessel sealing system, bipolar electrosurgery, harmonic scalpel, titanium clips, endoscopic gastrointestinal anastomosis vascular staples and sutures for arterial and venous ligation in a porcine model. J Urol. 2003; 169(2): 697-700, doi: 10.1097/01.ju.0000045160.87700.32, indexed in Pubmed: 12544345 .

9. Kennedy JS, Stranahan PL, Taylor KD, et al. High-burst-strength, feedback-controlled bipolar vessel sealing. Surg Endosc. 1998; 12(6): 876-878, doi: 10.1007/s004649900733, indexed in Pubmed: 9602010.

10. Goldstein SL, Harold KL, Lentzner A, et al. Comparison of thermal spread after ureteral ligation with the Laparo-Sonic ultrasonic shears and the Ligasure system. J Laparoendosc Adv Surg Tech A. 2002; 12(1): 61-63, doi: 10.1089/109264202753486957, indexed in Pubmed: 11905864.

11. Demirturk F, Aytan H, Caliskan AC. Comparison of the use of electrothermal bipolar vessel sealer with harmonic scalpel in total laparoscopic hysterectomy. J Obstet Gynaecol Res. 2007; 33(3): 341-345, doi: 10.1111/j.1 447-0756.2007.00533.x, indexed in Pubmed: 17578364.

12. Hagen B, Eriksson N, Sundset M. Randomised controlled trial of LigaSure versus conventional suture ligature for abdominal hysterectomy. BJOG. 2005; 112(7): 968-970, doi: 10.1111/j.1471-0528.2005.00561.x, indexed in Pubmed: 15958001.

13. Heniford BT, Matthews BD, Sing RF, et al. Initial results with an electrothermal bipolar vessel sealer. Surg Endosc. 2001; 15(8): 799-801, doi: 10.1007/s004640080025, indexed in Pubmed: 11443443.

14. Crawford ED. Use of the LigaSure vessel sealing system in urologic cancer surgery. Grand Rounds Urology. 1999; 1: 10-17.

15. Guerrieri M, Sanctis A, Baldarelli M, et al. Electrothermal bipolar energy-based device in laparoscopic right colectomy: our experience. Minerva Chir. 2008; 63(6): 455-460, indexed in Pubmed: 19078877.

16. Janssen PF, Brölmann HAM, van Kesteren PJM, et al. Perioperative outcomes using LigaSure compared with conventional bipolar instruments in laparoscopic hysterectomy: a randomised controlled trial. BJOG. 2011; 118(13): 1568-1575, doi: 10.1111/j.1471-0528.2011.03089.x, indexed in Pubmed: 21895949.

17. Bessa SS. Ligasure vs. conventional diathermy in excisional hemorrhoidectomy: a prospective, randomized study. Dis Colon Rectum. 2008; 51(6): 940-944, doi: 10.1007/s10350-008-9214-7, indexed in Pubmed: 18273670.

18. Taşkın S, Şükür YE, Altın D, et al. Bipolar Energy Instruments in Laparoscopic Uterine Cancer Surgery: A Randomized Study. J Laparoendosc Adv Surg Tech A. 2018; 28(6): 645-649, doi: 10.1089/lap.2017.0639, indexed in Pubmed: 29323616. 
19. Cho HY, Choi KJ, Lee YL, et al. Comparison of two bipolar systems in laparoscopic hysterectomy. JSLS. 2012; 16(3): 456-460, doi: 10.4293/1 08680812X13462882736259, indexed in Pubmed: 23318073.

20. Wang JY, Lu CY, Tsai HL, et al. Randomized controlled trial of LigaSure with submucosal dissection versus Ferguson hemorrhoidectomy for prolapsed hemorrhoids. World J Surg. 2006; 30(3): 462-466, doi: 10.1007/s00268-005-0297-1, indexed in Pubmed: 16479346.

21. Seehofer D, MoglM, Boas-Knoop S, et al. Safety and efficacy of new integrated bipolar and ultrasonic scissors compared to conventional laparoscopic 5-mm sealing and cutting instruments. Surg Endosc. 2012; 26(9): 2541-2549, doi: 10.1007/s00464-012-2229-0, indexed in Pubmed: 22447285.
22. Garry R, Fountain J, Mason Su, et al. The eVALuate study: two paralle randomised trials, one comparing laparoscopic with abdominal hysterectomy, the other comparing laparoscopic with vaginal hysterectomy. BMJ. 2004; 328(7432): 129, doi: 10.1136/bmj.37984.623889.F6, indexed in Pubmed: 14711749.

23. Rothmund R, Kraemer B, Brucker S, et al. Laparoscopic supracervical hysterectomy using EnSeal vs standard bipolar coagulation technique: randomized controlled trial. J Minim Invasive Gynecol. 2013; 20(5): 661-666, doi: 10.1016/j.jmig.2013.04.014, indexed in Pubmed: 23791399. 